\title{
GLAUCOMA, DEPRESSION AND QUALITY OF LIFE: MULTIPLE COMORBIDITIES, MULTIPLE ASSESSMENTS AND MULTIDISCIPLINARY PLAN TREATMENT
}

\author{
Goran Pelčići ${ }^{1}$, Rudolf Ljubičić ${ }^{2}$, Josip Barać ${ }^{3}$, Dubravka Biuk ${ }^{3} \&$ Veljko Rogoić ${ }^{4}$ \\ ${ }^{1}$ Department of Ophthalmology, Faculty of Medicine, University of Rijeka and Clinical Hospital Center Rijeka, \\ Rijeka, Croatia \\ ${ }^{2}$ Institute of Public Health of Primorsko-Goranska County, Rijeka, Croatia \\ ${ }^{3}$ Department of Ophthalmology and Optometry, Faculty of Medicine, \\ University J.J. Strossmayer of Osijek and Clinical Hospital Center Osijek, Osijek, Croatia \\ ${ }^{4}$ Department of Ophthalmology, Faculty of Medicine, University of Split \\ and Clinical Hospital Center Split, Split, Croatia
}

received: 17.8.2016;

revised: 9.3.2017;

accepted: 4.4.2017

\section{SUMMARY}

Background: The present mini review evaluates assessment and comorbidity of glaucoma and depression with the possibilities of treatment options.

Subjects and methods: Web of science was searched for relevant articles using search terms "glaucoma", "depression", "glaucoma and depression", "quality of life and glaucoma". The additional relevant papers were added from the bibliography of selected papers. All types of articles were included, no time period was defined.

Results: We have reviewed 11 studies regarding the comorbidities of glaucoma and depression. The number of participants in all studies ranged from 86 to 6760. Formats of scale in one study was clinician rated, others were self-reported scales. Two studies of the above mentioned studies explored specific types of glaucoma. In the three studies out of 11 there was no significant evidence of elevated depressive symptoms associated with glaucoma. In the six studies the prevalence of depression was significant among glaucoma patients. One study provided suboptimal assessments of depression in glaucoma patients. The other one showed the presence of glaucoma significantly associated with depression after adjustment for the demographic factor. Most of the studies used one scale, while two of them used two scales. The reviewed studies did not analyze the therapy options of included patients.

Conclusion: The results presented in this review indicate that glaucoma is accompanied by depression in the majority of analyzed studies. The comorbidity of glaucoma and depression should be the subject of further research on both, self-reflecting and clinician-rated scales, taking into account subjective experience of patients and physicians. The therapy options should be taken into account in future researches focusing on the multidisciplinary approach including novel possibilities of treating both diseases respectively.

Key words: glaucoma - depression - comorbidities - scales

$* * * * *$

\section{Introduction}

Glaucoma is a chronic, progressive, heterogeneous optic neuropathy which leads to visual field loss, disabilities and irreversible blindness if not diagnosed and treated at the right time. Glaucoma firstly affects peripheral vision and secondly central vision. Since it is silent, chronic disease with no clinical signs at the beginning of illness, it is often undiagnosed and underestimated (Eramudugolla et al. 2013). In many cases when diagnosed, glaucoma is already in advanced stage. Clinical trials confirmed the importance of intraocular pressure (IOP) in the development and progression of open-angle glaucoma, even if evidence suggests the existence of ocular and systemic factors, in addition to IOP, that can be responsible for this development and progression (Actis et al. 2016). These various risk factors may be organized into various categories (Agorastos \& Huber 2011, Nageeb \& Kulkarni 2015): demographic (age, sex and race), lifestyle (smoking, alcohol), comorbidities (heart disease, peripheral hypertension, diabetes, obesity), ophthalmic risk factors (eye structure, central corneal thickness, corneal hysteresis, intraocular pressure, myopia, cataract, vascular abnormalities), family history, socioeconomic status and compliance (Salowe et al. 2015). The point is that the main risk factor is ocular pressure (it is also the only one we can treat today), then we can discuss upon the others. In general, patients with visual impairment have higher prevalence of psychological disturbance - such as depression, anxiety or sleep disorders (Agorastos et al. 2013, Eramudugolla et al. 2013, Rovner et al. 2014, Skalicky \& Goldberg 2008). It was shown that the strongest connection between psychological disturbances and visual impairment disorder was depression (McCusker \& Koola 2015, Actis et al. 2016, Spinelli et al. 1998). The level of depressive symptoms is higher in patients with multiple eye disease (Eramudugolla et al. 2013, Pop-Jordanova et al. 2014). Dual sensory loss, such as visual and hearing impairment, increases even more the level of depressive symptoms (Kiely et al. 2013). On the other hand, depression is one of the most 
common psychological disturbances among patients that is comorbid with other chronic physical diseases in general (Jeong et al. 2016, Moussavi et al. 2007) with the most negative effect on the health score. Depression has also higher prevalence among older population (Skalicky \& Goldberg 2008). The risk factors for the development of psychological disorders in glaucoma patients could be subjective and objective (Kong et al. 2014). The age, sex, visual field loss (VFL) and the severity of glaucoma condition are some of the objective risk factors on which the patients have no influence (Kong et al. 2014). The subjective risk factors, such as regular and proper administration of topical antiglaucoma medication, education and proper understanding of character of the glaucoma disease or adoption of healthy habits, could be influenced by patients. In the study among the Chinese patients, the comprehension of the character of glaucoma is an independent factor negatively related to psychological disturbance and positively related to the Quality of Life (QoL) among the glaucoma patients (Kong et al. 2014). The authors emphasize the value of education in establishing the healthy habits (the importance of compliance with the therapy) among the patients. The knowledge of glaucoma character with consequent understanding of the value of therapy compliance decreased psychological disturbance and contributed to the higher QoL. In the paper of Agorastos et al, visual field defects (VFD) were predictive factors of depression, anxiety and sleep disorder (Agorastos et al. 2013). The loss of everyday life abilities (driving the car, reading, moving indoors and outdoors, depending on the help of others) could be an important factor in developing depression in glaucoma patient.

\section{Methodology}

The literature search was performed by using PubMed and MEDLINE. The searched terms were "glaucoma", "depression", "glaucoma and depression", "quality of life and glaucoma". This mini review was limited to the articles published in English language. All types of relevant articles were included in the review, including the original research, reviews and other articles of interest. The additional relevant papers were added from the bibliography of selected paper. The time period was not defined.

\section{Pathophysiology of glaucoma and depression}

Ophthalmic risk factors for glaucoma onset and development are: first of all intraocular pressure (IOP), followed by eye structure (angle of the anterior chamber), central corneal thickness, myopia, cataract, and vascular abnormalities (Salowe 2015). The important pathophysiological mechanism of glaucoma is redundant retinal oxidative stress (ROS) with the consequence on glaucomatous changes and neurodegeneration. The result is the loss of the intrinsically photosensitive retinal ganglion cells (ipRGC) with consequent disorder in melanopsin activity (Agorastos \& Huber 2011), disruption of circadian rhythm and neuropathy of optic nerve (Agorastos et al. 2013, Agorastos \& Huber 2011, Ayaki et al. 2015). Consequently, the role of neurohormone melatonin (MLT) in the eye may be involved in the physiology and pathophysiology of retinal function (Agorastos \& Huber 2011, Tosini et al. 2012). MLT has a potential use in the management of glaucoma (Agorastos \& Huber 2011) as protector against oxidative stress in neurons, antioxidant in the retina, with direct effect on intraocular pressure (IOP) and interference on other glaucomatous risk factors (hypertension, diabetes).

MLT has also an important role in the regulation of circadian rhythm by modulating electrical activities of neurons of suprachiasmatic nucleus. Changes in circadian system or chronodisruption can have an effect on sleep disturbance, seasonal affective disorders and depressive disorders (Tosini 2012). Pathophysiology of depression is not unique and clearly defined. It was shown that serotonin (Dell'Osso 2016), norepinephrine, glutamate and dopamine have an important role in pathophysiology of depressive disorders (Niciu et al. 2014). Recent researches indicate that MLT might have an important role in pathophysiology and possible therapy in many diseases, such as depression, diabetes, pain, neurodegenerative diseases, circadian and mood disorders, insomnia (Comai \& Gobbi 2014), and vision disorder such as glaucoma (Agorastos \& Huber 2011, Tosini 2012). MLT is synthesized from serotonin and acts through MT1 and MT2 receptors (Agorastos \& Huber 2011, Comai 2014). In the study of Comai and Gobi MT2 receptors were involved in the pathophysiology and pharmacology of depression (Comai \& Gobbi 2014). In addition, MT1 and MT2 receptors were identified in mammalian retina (Tosini et al. 2012). Therefore, MLT might be connected with reducing of IOP (Tosini et al. 2012). Pathophysiological connection between depression and glaucoma might origin from the reduced absorption of light because of retinal disruption which leads to irregular melatonin synthesis as it was showed in various animal models (Tosini et al. 2012). The result is higher prevalence of depression, anxiety, and sleep disorder.

There is no clear evidence of effectiveness of neuroprotective agents on progression of OAG (Sena \& Lindlsey 2017). Further clinical researche should plan to explore the benefits of neuroprotective agents on OAG and preservation of vision.

\section{Epidemiological data}

The epidemiological studies indicated an increase in the prevalence of glaucoma (Vost et al. 2015, Boyers et al. 2015, Ferrari et al. 2010, Tham et al. 2014, Wang et al. 2012). In 2013, 64,3 million people with glaucoma (aged between 40 and 80 years) were estimated. It is 
calculated that the number of people with glaucoma will have increased up to 76,0 million by the year 2020 and up to 111,8 million by the year 2040 respectively (Tham et al. 2014). Since glaucoma affects mostly older population, whose prevalence has been significantly increased (Skalicky et al. 2015), it presents one of the global public health issues (Tsai et al. 2004, Chen et 1 2015). Global Burden of Disease Study 2013 had ranked the glaucoma prevalence with total percentage of $1.29 \%$ in $1990,1.41 \%$ in 2013 , while the YLD (years lived with disabilities) ranked $3,58 \%$ in 1990 , and $4.02 \%$ in 2013 . Glaucoma prevalence by severity was ranked in the same study in 2013; moderate $0.94 \%$, severe $0.13 \%$ and blind $0.34 \%$ (Vost et al. 2015). The same study indicated significant percentage change in prevalence of glaucoma from 1990 to 2013 (50.2\% vs $74.7 \%)$ respectively, the significant changes are YLD from 1990 to $2013(54.8 \%$ vs $70.9 \%)$ and in agestandardized YLD from 1990 to 2013 (-9.7\% vs $-0.2 \%)$ (Vost et al. 2015). Glaucoma and macular degeneration global burden were overrepresented according to the Global burden of eye and vision disease as reflected in the Cochrane Database of Systematic Reviews (Boyers et al. 2015). This finding might suggest that certain diseases such as glaucoma attract much more attention of researchers than others, because of their importance in causing morbidity and disabilities.

The results of Moussavis' research, World Health Study (WHS) by World Health Organization (WHO), showed that one-year prevalence of depressive episode was $3.2 \%$ in the 60 countries involved in research (Moussavi et al. 2007). In the case of comorbid depression (depression with chronic physical disease) the prevalence was significantly higher, between $9.3 \%$ and $23.0 \%$. Depressive disorders were the second leading cause of YLD according to Global Burden of Disease Study 2010 (Ferrari et al. 2013) and 2013 (Vost et al. 2015, Global Burden 2013, Kessler \& Bromet 2013).

According to the findings of several studies, (Wang et al. 2012, Mabuchi et al. 2012, Skalicky \& Goldberg 2008) which were undertaken worldwide, the prevalence of depression among glaucoma coincides. The prevalence of depression among glaucoma patients in the study of Wang et al. was $10.9 \%$ (Wang et al. 2012) as it was in the Japanese Clinical study (Mabuchi et al. 2012). The range of the prevalence of depression in glaucoma patients was between $11.4 \%$ and $32.1 \%$ in the Australian clinical study performed by Skalicky et and Goldberg (Skalicky \& Goldberg 2008).

\section{The quality of life of glaucoma patients with depression}

The concept of QoL in the last decades has become the focus of interest of many physicians, especially those who treat the chronic disease patients (Labiris et al. 2011). To comprehend the concept of QoL and its purpose in diagnosing and treatment of patients, the re- searchers need to be aware of the parameters that comprise it: physical, functional, social, and psychological (Labiris et al. 2011). The comorbidity of depression and glaucoma interfere with all of them (Moussavi et al. 2007, Skalicky \& Goldberg 2008). In the practice there are several instruments available (Severn et al. 2008, Labiris et al. 2011, Waisbourd et al. 2015, Sprat et al. 2008, Valderes et al. 2004, Brown et al. 1998) for the assessment of the QoL of glaucoma patients that can be divided in three groups: Health related QoL, vision-specific QoL, and disease specific QoL - glaucoma specific instruments. Glaucoma specific tools have higher specificity for glaucoma. According to the literature (Labiris et al. 2011, Severn et al. 2008, Spratt et al. 2008), there are no ideal or generally used Qol instruments for glaucoma. But regardless of that, most of the conducted studies showed that glaucoma patients have lower Qol scores and less functional status (Tsai et al. 2003, van Gestel et al. 2010, Wilson et al. 1998). It was shown that the visual impairment limits the QoL of patients (Skalicky et al. 2015, Labiris et al. 2011). The depression comorbid with chronic conditions such as glaucoma worsen the health status of patients (Moussavi et al. 2007) and have negative implication on the QoL. It was shown that the age related eye diseases like glaucoma are associated with poorer health related quality of life (HRQoL) ( $\mathrm{Li}$ et al. 2011, Tsai et al. 2004) by worsening poor general health, physical and mental unhealthy days such as limitation of daily activities. Contrary, HRQoL did not deteriorate in glaucoma patients in the study of Esteban et al. (Esteban et al. 2008). Deterioration of glaucoma with VFL is associated with the loss in glaucomaspecific QoL and general health QoL (van Gestel et al. 2010). As the last study indicated, two types of QoL instruments (general health and glaucoma-specific instruments) might be used to assess the proper Qol in glaucoma patients.

\section{Assessment of depression in glaucoma patients}

The analyzed literature in this paper provides an overview of instruments used for assessment of depression in glaucoma patients (Gothwal et al. 2014, Cumurcu et al. 2006, Skalicky \& Goldberg 2008, Wang et al. 2012, Ayaki et al. 2015, Kiely et al. 2013, Eramudugolla et al. 2013, Wilson et al. 2002, Agorastos et al. 2013, Kong et al. 2014). The screening of the depression like disorder, diagnose of the disorder, assessment of severity of disorder, and the measurement of changes in severity of disorders represent the main purpose of assessment of the scales for depression (Furukawa 2010). The psychiatric assessments can be organized in two formats: clinician-rated and self-report (Furukawa 2010).

Hamilton Depression Rating Scale (HDRS) is clinician-rated scale with 21 items for assessing the severity and measuring the changes of depression (Furukawa 2010, Cumurcu et al. 2014). 
Table 1. The comparison between reviewed studies based on the assessment of depression, format of rating scales for depression, format of glaucoma assessment and total number of participants

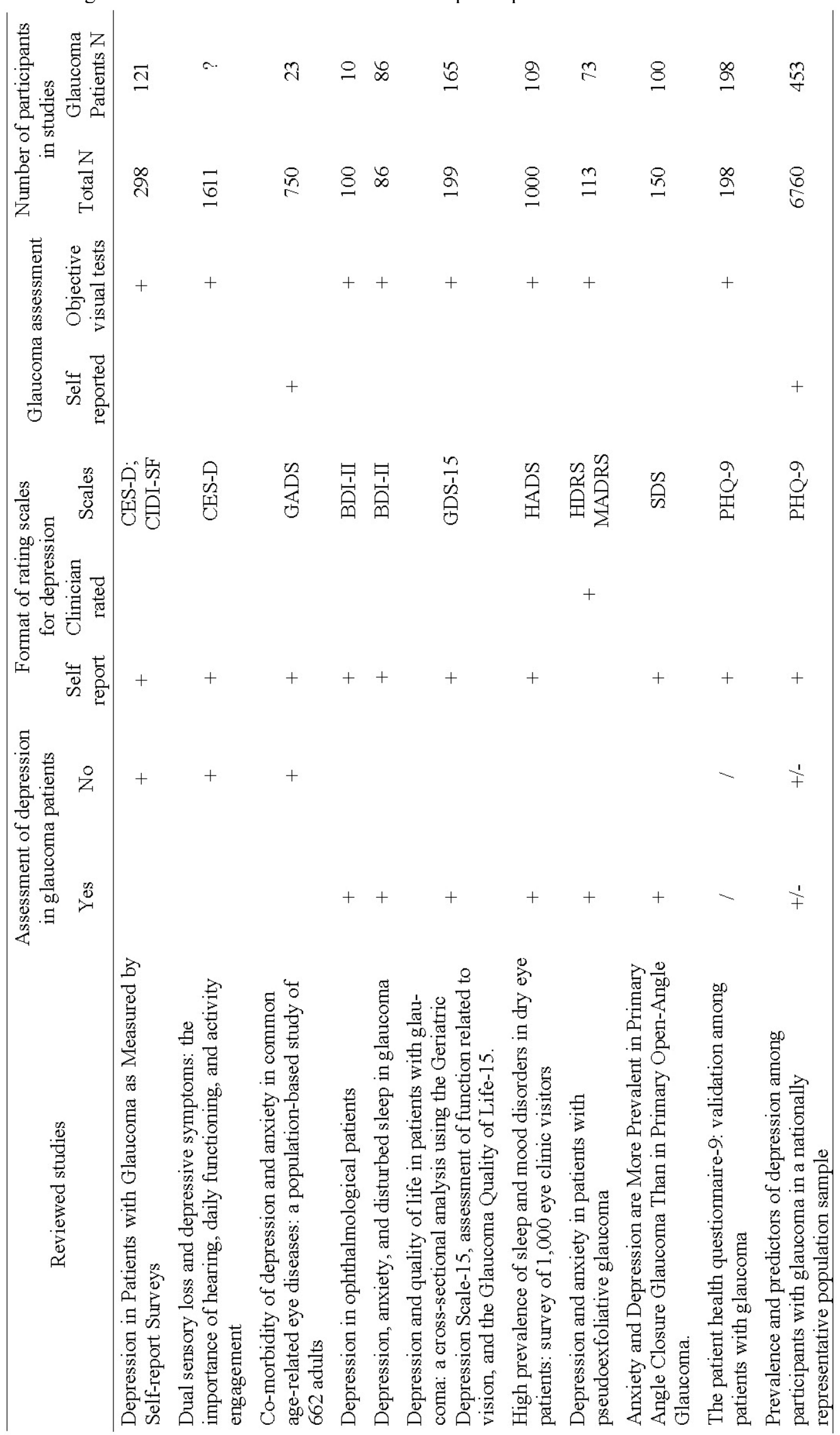


Montgomeri-Asberg Depression Rating Scale (MADRS) is clinician-rated instrument created with the purpose of measuring the severity and changes of depression (Furukawa 2010, Cumurcu et al. 2006).

Center for Epidemiological Studies Depression Scale (CES-D) is 20 item, self-repot instrument which responses are based on depression symptoms experienced one week before the testing. It is used for epidemiological studies of depression in population (Wilson et al. 2002, Kiely et al. 2013).

Composite International Diagnostic Interview-Short Form (CIDI-SF) scale was originally constructed by WHO for assessment of mental disorder. CIDI-SF assesses 8 of 9 symptoms for diagnosing the depressive episode (criteria of Diagnostic and Statistical Manual of Mental Disorder, $4^{\text {th }}$ Edition of American Psychiatric Association) (Gigantesco \& Morosini 2008, Wilson et al. 2002).

Hospital Anxiety and Depression Scale (HADS) is self-report, created to detect anxiety and depression. It is broadly used in hospital-based surveys, (Kong et al. 2015, Ayaki et al.). It contains two subscales (depression and anxiety), both with 7 items. The answers are based on the relative frequency of symptoms over the past week.

Beck's Depression Inventory-Second Edition (BDIII) is 21 item self-report and possible self-scored scale for screening of severity and measuring of changes in depression (Furukawa 2010, Agorastos et al. 2013, PopJordanova et al. 2014).

Goldberg Anxiety and Depression Scales (GADS) is self-completed, 18 item questionnaire designed for measurements of the anxiety and depression ( 9 anxiety and 9 depression items) (Koloski et al. 2008g, Eramudugolla et al. 2013).

Patient-Health Questionnaire (PHQ)-9 is 9 item, self-report version of Primary Care Evaluation of Mental Disorders (PRIME-MD) developed in 1999 (Furukawa 2010, Wang et al. 2012, Gotwall et al. 2014). The purpose of PHQ-9 is self-reported screening of depression, measuring of severity and measuring of changes in depression. The responds are ranked based on the frequency of depressive symptoms over the previous two weeks. In the analyses of validity for screening PHQ-9 showed substantial heterogeneity which was not explained (Furukawa 2010). PHQ-9 provided suboptimal assessment of depression in glaucoma patients in India, in Study of Gothwal et al. (Gotwall et al. 2014).

Geriatric Depression Scale-15 (GDS-15) is selfreport instrument with 15 items used to assess depression in elderly population (Skalicky \& Goldberg 2008).

Self-rating Depression Scale (SDS) is 20 item, selfreported scale, used to asses affective, psychological and somatic symptoms associated with depression. The responses are ranked according to the frequency of symptoms in the past several days (Kong et al. 2015).

\section{Depression and glaucoma - studies}

On the basis of the above mentioned scales, 11 studies were extracted in this review. The prevalence of depression in patients with visual impairment is the issue of research in many studies (Ayaki et al. 2015, Eramudugolla et al. 2013, Wang et al. 2012, Skalicky \& Goldberg 2008). For example, in four of the extracted studies the prevalence of depression in glaucoma patients was significantly higher (Ayaki et al. 2015, PopJordanova et al. 2014, Agorastos et al. 2013, Skalicky \& Goldberg 2008). The HADS score for depression was the highest in glaucoma patients in comparison to other patients with visual impairment in the study of Ayaki et al. (Ayaki et al. 2015). This study was primary designed for the assessment of prevalence of sleep and mood disorders in the eye clinic patients. The total number of participants was 730, only 109 of them were glaucoma patients (e.g. Table 1). The severity of glaucoma and older age of the patients, in the study of Skalicky and Goldberg, contributed to higher prevalence of depression in glaucoma patients (Skalicky \& Goldberg 2008). The purpose of this study was also to determinate the association of depression and the QoL in glaucoma patients. Glaucoma specific instrument Glaucoma Quality of life-15 (GQL-15) was used for the QoL assessment. The results showed decreased quality of life with worsening severity of glaucoma. The total number and share of glaucoma patients are shown in the table 1 . The increasing trend of depression in this study might be due to decrease of central and peripheral vision with consequent reduction of subjective and objective visual function. In the study of Agorastos et al, the VFL in glaucoma was the principal predictive factor of depression (Agorastos et al. 2013) and other psychological disturbances. One of the main messages of this study is the necessity of screening for psychological disturbance and psychochronobiological treatment of glaucoma patients. The importance of regular screening for depression in glaucoma patients supports also the findings of the study of Pop-Jordanova et al, which show that depression was often unrecognized and untreated problem (Pop-Jordanova et al. 2014). The prevalence of depression was often higher in the patients with severe ophthalmological disturbances (glaucoma, age-related macular degeneration, proliferative diabetic retinopathy and cataract) in the study of Pop-Jordanova (PopJordanova et al. 2014) which is consistent with the study of Skalicky and Goldberg (Skalicky \& Goldberg 2008). In addition the level of depression was found higher in the elderly population which is in coincidence with both the above mentioned studies (Skalicky \& Goldberg 2008, Pop-Jordanova et al. 2014).

The two papers from this review reported comorbidity of specific type of glaucoma and depression (Cumurcu et al. 2006, Kong et al. 2015). The study of Cumurcu et al, discussed the association of pseudoexfoliative glaucoma (PXG) and depression (Cumurcu et al. 2006). The PXG patients were more depressed then 
patients with primary open angle glaucoma (POAG) and control group. The same study showed no significant differences in the anxiety scale comparing PXG or POAG group respectively, and the control groups. The higher level of depression was associated with the patients with primary angle closer glaucoma (PACG) in contrast to patients with POAG and control group in the study of Kong et al. (Kong et al. 2015). Both studies compared POAG with other types of glaucoma and both studies showed that patients with POAG were less depressed. However, on the basis of these two studies, it could not be possible to conclude that POAG patients are less depressed than patients who suffer from other types of glaucoma. The existence of association of certain type of glaucoma and depression (Cumurcu et al. 2006, Kong et al. 2015) should be evaluated in further studies.

In the three studies (Eramudugolla et al. 2013, Wilson et al. 2002, Kiely et al. 2013), there were no significant correlation between depression and glaucoma. Eramudugolla et al. associated such findings with selfreported knowledge of glaucoma that might have compromised the result because glaucoma is often underdiagnosed in population (Eramudugolla et al. 2013), which is consistent with the previous study of Pop-Jordanova (Pop-Jordanova et al. 2014). The same indicates the study of Kiely et al, e.g. there is no increase of depression in patients with only visual field loss (corrected distance visual acuity $>0,3$ longMar in the better eye, self-reported blindness and glaucoma) (Kiely et al. 2013). The glaucoma patients did not report higher depression than patients without glaucoma in the study of Wilson et al. (2002).

Finally, the last two studies from this review, which used PGQ-9 scale, did not have congruent results (Wang et al. 2012, Gothwal et al. 2014). PHQ-9 instrument in the study of Gothwal et al. with 198 patients did not provide optimal assessment of depression in glaucoma patients (Gothwal et al. 2014). In the study of Wang et al. (2012), where the PHQ-9 scale was also used, the presence of correlation between depression and glaucoma had no significance without the adjustment. The authors used two models of adjustments: adjustments for demographic factors and for the selfreport of the general health condition. The model in which glaucoma and depression were adjusted for the demographic factors, the association of glaucoma and depression, was significant. The model with adjustment for the general health condition found no significant correlation with glaucoma and depression. We can calculate that PHQ-9 is not the instrument of choice for the assessment of depression in glaucoma patients. On the other hand, Furukawa reported (Furukawa 2010) PHQ-9 as an instrument with excellent psychometric properties but with substantial heterogeneity in screening validity, which could not be explained.

There are no unique results of the studies regarding the association of depression with glaucoma (McCusker \& Koola 2015, Kiely et al. 2013, Wilson et al. 2002,
Tosini et al. 2012, Eramudugola et al. 2013, Skalicky \& Goldberg 2008) using different measurement instruments (e.g. Table 1). Table 1 recapitulates the comparison between reviewed studies based on the assessment of depression, format of rating scales for depression, format of glaucoma assessment and the total number of the participants with the share of glaucoma patients (e.g. Table 1). Only one study used clinician-rated scales (HDRS and MADRS) for the assessment of depression in glaucoma patients (Cumurcu et al. 2006). Most of the studies used objective visual tests for glaucoma assessment. Only in two studies glaucoma was assessed by self-report method (Ermudugolla et al. 2013, Wang et al. 2012). In the first study, there was no evidence for elevated rates of depression in glaucoma (Ermudugolla et al. 2013). The total number of participants and the number of glaucoma patients varied widely among reviewed studies (e.g. Table 1). The total number of participants ranged from 6760 to 86 . The range of glaucoma patients varied from 10 to 453 . In one study the number of glaucoma patients was unknown (Kiely et al. 2013). Some of the studies were performed only with the glaucoma patients (Gothwal et al. 2014, Agorastos et al. 2013), while others had combination of patients with visual impairment, control group. One of the issues which could have influenced the results was the range of glaucoma patients in the study.

\section{Management of patients who suffer from glaucoma and depression}

The aging of population, the increase in various comorbidities, the various available medications and the organization of health care pose a great challenge for clinicians in the management of chronic patients today.

By focusing only on the treatment of glaucoma, comorbidities are often neglected. Depression as a comorbid condition deteriorates the health of the patients more than depression alone or more than other chronic conditions, alone or in combination of different chronic diseases without depression (Moussavi et al. 2007). Chronic conditions such as glaucoma may be associated with depression due to the age of patients (Jeong et al. 2006, Kong et al. 2014), prolonged daily use of antiglaucoma medications, limitation of daily activities, limitation of family and working roles (Guedes et al. 2012).

The antiglaucoma medication is mostly topical application. The possibilities of systematic adverse effects are uncommon but they are still possible (Jeong et al. 2016). For example, the caution with regard to use of ocular beta-blockers was recommended in the case of depression, asthma and heart disease. However, recent studies did not find any correlation between beta-blocker use and depression (Wilson et al. 2002). The recent studies have also suggested that phenylephrine eye drops had no clinically relevant effects on the heart rate or blood pressure as was previously suggested (Stavert et al. 2015). 
The topical administration of antiglaucoma medications, on the other hand, has very common topical side effects (Guedes et al. 2012, Servat \& Bernardino 2011, Park et al. 2013) such as eyelid dermatitis, lacrimal system scarring, ocular discomfort upon instillation, conjunctival inflammation and conjunctival epithelium changes, corneal surface and endothelial impairment, blurred vision, a stinging sensation etc. The patients diagnosed with glaucoma during the period when the symptoms of visual field and visual function loss had not still been developed, could only experience the disturbing side effects of antiglaucoma therapy. As a result, some of the early diagnosed glaucoma patients might give up on therapy. The initial education of patients regarding the possibilities of side effects of the antiglaucoma therapy and the severe consequences of nonadherence to it is very important (Park et al. 2013).

The price of medications, the difficulties with administration and dosing schedule might contribute to poor compliance, worsening IOP, and consequently depression. The patient is in vicious circle.

The different situation with the same result appears in patients with good compliance, sometimes multiple ophthalmological therapy (surgery and multiple medications), regular follow-ups, with the result of advanced glaucoma and VFL with the respond of depression (Gothwal et al. 2014).

Administration of psychiatric medication can cause glaucoma (Jeong et al. 2016). Tricyclic antidepressants can lead to closed-angle glaucoma because of anticholinergic activity. Because of epinephrine receptor blockade, some of the antipsychotic drugs can also cause closed-angle glaucoma. Selective serotonin inhibitors and benzodiazepines can generate in closed-angle glaucoma. Long-term use of selective serotonin reuptake inhibitors (SSRI), which are the most prescribed type of medication for depression, does not significantly influence the risk of POAG and PACG (Chen et al. 2015). One of the future possibilities in the management of comorbidity of glaucoma and depression is MLT, as it was described in the papers of Agorastos and Huber (Agorastos \& Huber 2010), and Tosini et al. (Tosini 2012).

The character of depression with the hopelessness and pessimism may cause poor compliance and withdrawal of the therapy (Jeong et al. 2016).

Besides the standard therapy of glaucoma, topical antiglaucoma medications and surgery, novel approaches such as melatonin (Agorastos \& Huber 2011, Tosini et al. 2012), endocannabinoid system (Cairns et al. 2016), and bone-marrow mesenchymal stem cell (Roubeix et al. 2015) should be explored.

Cognitive deficits are often comorbid with depression (Joeng et al. 2016). Due to cognitive deficit the patients are not able to comprehend the character of glaucoma and the necessity of adherence to the therapy which leads to worsening of glaucoma and blindness.
The management of the patients with comorbidity of glaucoma and depression should be organized on the basis of a multidisciplinary approach (Jeong et al. 2016). An ophthalmologist has to recognize and take into account a state of depression when the treatment plan is made (Moussavi et al. 2007).

The organization of health care in the case of subspecialist glaucoma ambulance is an important factor in the management of glaucoma patients (the time for the review; education about the characteristics of glaucoma, possibilities and side effects of treatment; the real role of glaucomatologyst, the role of a subspecialty nurse). The next step should be the involvement of a multidisciplinary team consisting of psychologists, psychiatrists and rehabilitation therapists (Rovner et al. 2014).

\section{Conclusion}

The results presented in this review indicated that glaucoma is accompanied by depression in the majority of analyzed studies. The rating scales used in assessing depression in studies analyzed in this review had different purposes and different formats. The questionable is connection of formats of instruments used in the studies (self-reporting or clinician-reporting) and assessments or no assessment of depression. Besides the issues of different instruments, formats of assessments and the number of participants in the studies, subjective experience of patients and physicians should be taken into account when assessing the depression in glaucoma patients (Wang et al. 2012). The reviewed studies did not analyze therapy options of glaucoma patients with depression. The treatment of comorbidity of glaucoma and depression should not depend only on ophthalmologist or psychiatrist, the assessment of treatment should be multidisciplinary. New approaches, such as MLT administration, should be taken into account in the management of glaucoma patients with depression. The management of glaucoma and depression should be comprehended as a public-health issue because of epidemiological data regarding the rising prevalence of both chronic diseases worldwide.

\section{Acknowledgements: None.}

\section{Conflict of interest: None to declare.}

\section{Contribution of individual authors:}

Goran Pelčić: concept and design of study, drafting the manuscript, critical revision for intellectual content, supervision;

Rudolf Ljubičić: drafting the manuscript, critical revision for intellectual content;

Josip Barać: literature searches and analyses, critical revision for intellectual content;

Dubravka Biuk: interpretation of data, critical revision for intellectual content;

Veljko Rogoić: critical revision for intellectual content, administrative and technical support. 


\section{References}

1. Actis AG, Versino E, Brogliatti B, Rolle T: Risk Factors for Primary Open Angle Glaucoma (POAG) Progression: A Study Ruled in Torino. Open Ophthalmol J 2016; 10:129-39.

2. Agorastos A \& Huber CG: The role of melatonin in glaucoma: implications concerning pathophysiological relevance and therapeutic potential. J Pineal Res 2011; 50:1-7.

3. Agorastos A, Skevas C, Matthaei M, Otte C, Klemm M, Richard $G$ et al: Depression, anxiety, and disturbed sleep in glaucoma. J Neuropsychiatry Clin Neurosci 2013; 25:205-13.

4. Ayaki M, Kawashima M, Negishi K, Tsubota K: High prevalence of sleep and mood disorders in dry eye patients: survey of 1,000 eye clinic visitors. Neuropsychiatr Dis Treat 2015; 31:11:889-94.

5. Boyers LN, Karimkhani C, Hilton J, Richheimer $W$, Dellavalle RP: Global burden of eye and vision disease as reflected in the Cochrane Database of Systematic Reviews. JAMA Ophthalmol 2015; 133:25-31.

6. Brown GC, Brown MM, Sharma S, Brown HC: Patient perceptions of quality-of-life associated with bilateral visual loss. Int Ophthalmol 1998; 22:307-12.

7. Cairns EA, Baldridge WH, Kelly ME: The Endocannabinoid System as a Therapeutic Target in Glaucoma. Neural Plast 2016; 2016:9364091.

8. Chen HY, Lin CL, Kao CH: Long-Term Use of Selective Serotonin Reuptake Inhibitors and Risk of Glaucoma in Depression Patients. Medicine (Baltimore) 2015; 94:e2041.

9. Comai $S$ \& Gobbi G: Unveiling the role of melatonin MT2 receptors in sleep, anxiety and other neuropsychiatric diseases: a novel target in psychopharmacology. $J$ Psychiatry Neurosci 2014; 39:6-21.

10. Cumurcu T, Cumurcu BE, Celikel FC, Etikan I: Depression and anxiety in patients with pseudoexfoliative glaucoma. Gen Hosp Psychiatry 2006; 28:509-15.

11. Dell'Osso L, Carmassi C, Mucci F, Marazziti D: Depression, Serotonin and Tryptophan. Curr Pharm Des 2016; 22:949-54.

12. Eramudugolla R, Wood J, Anstey KJ: Co-morbidity of depression and anxiety in common age-related eye diseases: a population-based study of 662 adults. Front Aging Neurosci 2013; 2:5:56.

13. Esteban NJJ, Martínez MS, Navalón PG, Serrano OP, Patiño JRC, Purón MEC et al: Visual impairment and quality of life: gender differences in the elderly in Cuenca, Spain. Qual Life Res 2008;17(1):37-45.

14. Ferrari AJ, Charlson FJ, Norman RE, Patten SB, Freedman G, Murray CJ: Whiteford HA. Burden of depressive disorders by country, sex, age, and year: findings from the global burden of disease study 2010. PLoS Med 2013;10(11):e1001547.

15. Furukawa TA. Assessment of mood: guides for clinicians. J Psychosom Res 2010; 68(6):581-9.

16. Gigantesco A \& Morosini P: Development, reliability and factor analysis of a self-administered questionnaire which originates from the World Health Organization's Composite International Diagnostic Interview - Short Form (CIDI-SF) for assessing mental disorders. Clin Pract Epidemiol Ment Health 2008: 10:4-8.

17. Gothwal VK, Bagga DK, Bharani S, Sumalini R, Reddy SP: The patient health questionnaire-9: validation among patients with glaucoma. PLoS One 2014; 7:9(7):e101295.
18. Guedes RAP, Guedes VMP, Freitas SM, Chaoubah A: Quality of life of glaucoma patients under medical therapy with different prostaglandins. Clin Ophthalmol 2012; 6:1749-53.

19. Jeong AR, Kim CY, Kang MH, Kim NR: Psychological Aspects of Glaucoma. J Nerv Ment Dis 2016; 204:217-20.

20. Kessler RC \& Bromet EJ: The epidemiology of depression across cultures. Annu Rev Public Health 2013; 34:119-138.

21. Kiely KM, Anstey KJ, Luszcz MA: Dual sensory loss and depressive symptoms: the importance of hearing, daily functioning, and activity engagement. Front Hum Neurosci 2013; 7:837.

22. Koloski NA, Smith N, Pachana NA, Dobson A: Performance of the Goldberg Anxiety and Depression Scale in older women. Age Ageing 2008; 37:464-7.

23. Kong X, Yan M, Sun X, Xiao Z: Anxiety and Depression are More Prevalent in Primary Angle Closure Glaucoma Than in Primary Open-Angle Glaucoma. J Glaucoma 2015;24(5):e57-63.

24. Kong XM, Zhu WQ, Hong JX, Sun XH: Is glaucoma comprehension associated with psychological disturbance and vision-related quality of life for patients with glaucoma? A cross-sectional study. BMJ Open 2014; 26:4(5):e004632.

25. Labiris G, Giarmoukakis A, Kozobolis VP: Quality of Life (QoL) in Glaucoma Patients. In Rumelt S (ed): Glaucoma - Basic and Clinical Concepts, 307-318. In Tech Europe, 2011. Available at http://cdn.intechopen.com/pdfswm/23827.pdf. assessed: 30.05.2015.20

26. Li Y, Crews JE, Elam-Evans LD, Fan AZ, Zhang X, Elliott $A F$ : Visual impairment and health-related quality of life among elderly adults with age-related eye diseases. Qual Life Res 2011; 20:845-52.

27. Mabuchi F, Yoshimura K, Kashiwagi K, Yamagata Z, Kanba S, Iijima $H$ et al.: Risk factors for anxiety and depression in patients with glaucoma. Br J Ophthalmol 2012; 96:821-5.

28. McCusker $S$ \& Koola MM: Association of Ophthalmologic Disorders and Depression in the Elderly: A Review of the Literature. Prim Care Companion CNS Disord 2015; 20:17.

29. Moussavi S, Chatterji S, Verdes E, Tandon A, Patel V, Ustun B: Depression, chronic diseases, and decrements in health: results from the World Health Surveys. Lancet 2007; 370:851-8.

30. Nageeb $N$ \& Kulkarni UD: Glaucoma Awareness and SelfCare Practices among the Health Professionals in a Medical College Hospital. J Clin Diagn Res 2015; 9:NC01-4.

31. Niciu MJ, Ionescu DF, Richards EM, Zarate CA Jr: Glutamate and its receptors in the pathophysiology and treatment of major depressive disorder. $J$ Neural Transm (Vienna) 2014;121:907-24.

32. Park MH, Kang KD, Moon J: Noncompliance with glaucoma medication in Korean patients: a multicenter qualitative study. Jpn J Ophthalmol 2013;57(1):47-56.

33. Pop-Jordanova N, Ristova J, Loleska S: Depression in ophthalmological patients. Prilozi 2014,35(2):53-8.

34. Roubeix C, Godefroy D, Mias C, Sapienza A, Riancho L, Degardin $J$ et al: Intraocular pressure reduction and neuroprotection conferred by bone marrow-derived mesenchymal stem cells in an animal model of glaucoma. Stem Cell Res Ther 2015;6:177.

35. Rovner BW, Casten RJ, Hegel MT, Massof RW, Leiby BE, Ho AC. Low vision depression prevention trial in age- 
related macular degeneration: a randomized clinical trial. Ophthalmology 2014; 121:2204-11.

36. Salowe R, Salinas J, Farbman NH, Mohammed A, Warren JZ, Rhodes A: Primary Open-Angle Glaucoma in Individuals of African Descent: A Review of Risk Factors. $J$ Clin Exp Ophthalmol 2015; 6.

37. Sena DF, Lindsley K: Neuroprotection for treatment of glaucoma in adults. See comment in PubMed Commons belowCochrane Database Syst Rev 2017;1:CD006539. doi: 10.1002/14651858.CD006539.pub4.

38. Servat JJ, Bernardino CR: Effects of common topical antiglaucoma medications on the ocular surface, eyelids and periorbital tissue. See comment in PubMed Commons belowDrugs Aging 201;28:267-82.

39. Severn P, Fraser S, Finch T, May C: Which quality of life score is best for glaucoma patients and why? BMC Ophthalmol 2008; 8:2.

40. Skalicky $S$ \& Goldberg I: Depression and quality of life in patients with glaucoma: a cross-sectional analysis using the Geriatric Depression Scale-15, assessment of function related to vision, and the Glaucoma Quality of Life-15. J Glaucoma 2008; 17:546-51.

41. Skalicky SE, Fenwick E, Martin KR, Crowston J, Goldberg I, McCluskey P. Impact of age-related macular degeneration in patients with glaucoma: understanding the patients' perspective. Clin Experiment Ophthalmol 2015; [Epub ahead of print]

42. Spinal Cord Injury Research Evidence. Hospital Anxiety Depressive Scale (HADS). Available at: https://www.scireproject.com/outcome-measuresnew/hospital-anxiety-and-depression-scale-hads assessed:03.03.2016.

43. Spinelli D, Faroni E, Castellini G. The "personality" of the glaucomatous patient: preliminary results. Acta Ophthalmol Scand Suppl 1998; 227:53-4.

44. Spratt A, Kotecha A, Viswanathan A: Quality of Life in Glaucoma. JOCGP 2008; 2:39-45.

45. Stavert B, McGuinness MB, Harper CA, Guymer RH, Finger RP. Cardiovascular Adverse Effects of Phenylephrine Eyedrops: A Systematic Review and Meta-analysis. JAMA Ophthalmol 2015; 133:647-52.

46. Tham YC, Li X, Wong TY, Quigley HA, Aung T, Cheng $C Y$ : Global prevalence of glaucoma and projections of glaucoma burden through 2040: a systematic review and meta-analysis. Ophthalmology 2014;121:2081-90.
47. Tosini G, Baba K, Hwang CK, Iuvone PM: Melatonin: an underappreciated player in retinal physiology and pathophysiology. Exp Eye Res 2012;103:82-9.

48. Tosini G, Baba K, Hwang CK, Iuvone PM: Melatonin: an underappreciated player in retinal physiology and pathophysiology. Exp Eye Res 2012;103:82-9.

49. Tsai SY, Chi LY, Cheng CY, Hsu WM, Liu JH, Chou P: The impact of visual impairment and use of eye services on health-related quality of life among the elderly in Taiwan: the Shihpai Eye Study. Qual Life Res 2004;13:1415-24.

50. Valderas JM, Alonso J, Prieto L, Espallargues M, Castells $X$ : Content-based interpretation aids for health-related quality of life measures in clinical practice. An example for the visual function index (VF-14). Qual Life Res 2004; 13:35-44.

51. van Gestel A, Webers CAB, Beckers HJM, van Dongen $M C J M$, Severens JL, Hendrikse F: The relationship between visual field loss in glaucoma and health-related quality-of-life. Eye 2010; 24:1759-69.

52. Vos T, Barber RM, Bell B, Bertozzi-Villa A, Biryukov S, Bolliger I-Global Burden of Disease Study 2013 Collaborators: Global, regional, and national incidence, prevalence, and years lived with disability for 301 acute and chronic diseases and injuries in 188 countries, 19902013: a systematic analysis for the Global Burden of Disease Study 2013. Lancet 2015; 386:743-800.

53. Waisbourd M, Parker S, Ekici F, Martinez P, Murphy R, Scully $K:$ A prospective, longitudinal, observational cohort study examining how glaucoma affects quality of life an visually-related function over 4 years: design and methodology. BMC Ophtalmology 2015; 15:91.

54. Wang SY, Singh K, Lin SC: Prevalence and predictors of depression among participants with glaucoma in a nationally representative population sample. Am $J$ Ophthalmol 2012; 154:436-444.e2.

55. Wilson MR, Coleman AL, Yu F, Bing EG, Sasaki IF, Berlin $K$ et al.: Functional status and well-being in patients with glaucoma as measured by the Medical Outcomes Study Short Form-36 questionnaire. Ophthalmology 1998; 105:2112-6.

56. Wilson MR, Coleman AL, Yu F, Fong Sasaki I, Bing EG, Kim MH: Depression in patients with glaucoma as measured by self-report surveys. Ophthalmology 2002; 109:1018-22.

Correspondence:

Goran Pelčić, MD, assistant professor, ophthalmologist

Department of Ophthalmology, Faculty of Medicine

University of Rijeka and Clinical Hospital Center Rijeka

Braće Branchetta 20/1, 51000 Rijeka, Croatia

E-mail:goran.pelcic@medri.uniri.hr 